\title{
Signal Space Diversity for Improving the Reliability Performance of OFDM with Subcarrier Power Modulation
}

\author{
MOHAMMED HIJAZI ${ }^{1}$, AND JEHAD HAMAMREH ${ }^{2}$ \\ ${ }^{1}$ Electrical and Computer Engineering School, Antalya Bilim University, Antalya, Turkey. \\ ${ }^{2}$ Wislab, Department of Electrical-Electronics Engineering, Antalya Bilim University, Antalya, Turkey. \\ Corresponding author: Mohammed Hijazi.
}

The Matlab simulation codes used to generate the results in this paper can be found at https://researcherstore.com

\begin{abstract}
As the demand for higher data rates continues to increase exponentially, there is a shift towards exploring contemporary techniques that are likely to offer higher spectral efficiency. A new modulation technique termed as orthogonal frequency division multiplexing with sub-carrier power modulation (OFDM-SPM) has recently been introduced. It is an original technique that is still largely underexplored and aims at transmitting more bits per sub-carrier by manipulating the power of the sub-carriers in an OFDM block, in addition to those bits that are usually transmitted by conventional modulation schemes such as M-PSK. During the investigation and theoretical testing stages, it was found that the additional data stream conveyed by subcarriers' power has higher bit error rate (BER) performance compared to the data stream conveyed by conventional modulation schemes. To overcome this shortfall, signal space diversity (SSD) along with coordinate interleaving is proposed in this work to be integrated with OFDM-SPM to help improve the overall BER performance. By doing so, it is shown that a BER performance of $10^{-3}$ can be obtained at SNR of $15 \mathrm{~dB}$, which means achieving an improvement of more than $5 \mathrm{~dB}$ compared to the case of using OFDM-SPM without SSD. Furthermore, the performance results of OFDM-SPM-SSD is compared with those of OFDM-SPM with maximal ratio combining (MRC) and the obtained results show that OFDMSPM-SSD offers superior performance. Additionally, a study of the effect of the constellation rotation on OFDM-SPM is provided and an in-depth analysis is also carried out for different power polices of subcarrier power modulation with regards to two main performance metrics; namely BER and the throughput. Simulation results show that SSD provides a considerable improvement in the BER over both plain OFDMSPM and OFDM-SPM-MRC. The analysis also reveal that when considering the different power policies of SPM, the BER results are optimum in the case of using power-reassignment policy.
\end{abstract}

INDEX TERMS SSD, MRC, SNR, BER, OFDM, OFDM-SPM, Adaptive Modulation, Rayleigh Fading, Receive Diversity, Signal Space Diversity.

\section{INTRODUCTION}

W ITH the world moving towards the next wireless revolution and with the dramatic increase in the online access of the resources, there is a consistent need for increased data rates along with more spectrally efficient techniques for data transmission. With the advancement in wireless technology and the launch of $5 \mathrm{G}$ wireless communication, the motivation and the need for the development of spectrally efficient methods is evident.

Orthogonal frequency division multiplexing (OFDM) is a technique that is widely accepted for transmission over many wireless technologies [1]-[7]. OFDM allows the overlapping of sub-carriers while maintaining orthogonality, thereby improving the bandwidth efficiency. It also minimizes the adjacent channel interference and compensates for the multi-path wireless channel effects by dividing the incoming data stream into many parallel narrowband subchannels such that the narrowband subchannels become flat-faded and frequency independent fading channels.

In the present scenario, where the majority of people in the world are working online, the demand for higher data rates and the need for the development of new applications has 
increased in many fields. This provides a strong requirement for the further development of new techniques that improve spectral efficiency and provide higher data-rates with improved throughput.

OFDM with sub-carrier power modulation (OFDM-SPM) has recently been introduced and considered as a candidate that can provide a new dimension to OFDM thus resulting in an improved spectral efficiency [7]. In OFDM-SPM, the power of each sub-carrier is used to transmit extra bits along with the bits which are transmitted by conventional symbol modulation schemes in OFDM. In this way, it helps in improving the spectral efficiency along with an additional gain of reducing and savings the transmission power. In an effort to improve the performance, in [8], authors introduce a new non-coherent modulation scheme called orthogonal frequency division multiplexing with subcarrier power modulation and differential phase-shift keying (OFDM-SPMDPSK). The proposed technique has the potential to reduce complexity, where authors try to use the maximal ratio combining (MRC) to overcome the bit error degradation brought by the adoption of a non-coherent structure. In addition, the computational complexity is discussed.

In [9], the authors' presented performance results show that OFDM-SPM can be used with higher order modulation schemes while maintaining all the gains exhibited in OFDM-SPM with BPSK. This gives OFDM-SPM a unique advantage when compared to other 3D modulation schemes such as OFDM-IM and OFDM-SNM, which lose the gain in spectral efficiency as the modulation order becomes higher. Furthermore, the results of OFDM-SPM with QPSK were compared to that of conventional OFDM with 16-QAM symbol modulation. In [10], OFDM-SPM technique is investigated over a wireless multipath Rayleigh fading channel, where it introduces negligible complexity to the system, does not exhibit error propagation, reduces the transmission delay, and decreases the transmission power by half.

There are several types of diversity techniques that exist in the literature [11]-[16]. Signal space diversity (SSD) is one such diversity technique that has the potential to further improve the BER and spectral efficiency of a multi-carrier transmission system, such as OFDM. In SSD, a rotation is applied to the signal constellation of M-PSK/M-QAM such that the maximum number of data points have a distinguishable distance separating them even in the deepest noise at the receiver [14].

Moreover, coordinate interleaving and de-interleaving are also required in order to ensure that the in-phase (I) and Quadrature $(\mathrm{Q})$ components of the signal constellation are affected by independent fading. The authors in [15] discuss the coordinate interleaving as a possible method to exploit SSD with OFDM. The authors also derive the closed form expressions for the BER in a Rayleigh fading environment for implementing coordinate interleaving with OFDM. Additionally, the authors in [16], design the signal constellation code-book, for exploiting the SSD diversity technique with OFDM by grouping the sub-carriers. In [17], the authors analyze the optimum rotation parameters by employing SSD with simple QAM modulation. Spatial diversity in the receiver along with SSD and QAM is also studied. In [18], the authors apply rotation and coordinate interleaving on $\mathrm{M}$ ary phase shift keying (M-PSK) in Rayleigh fading channels. A closed-form expression for the upper-bound of the probability of bit error is derived and based on that, the optimum rotation angles are analyzed.

To the best of our knowledge, SSD with OFDM-SPM has not been explored yet. Hence, by exploiting the advantages of both OFDM-SPM and SSD, we can add a new dimension as well as an improvement to the BER and throughput of the OFDM-SPM. Higher throughput at low SNR is the main requirement of many low power IoT devices and WSNs. To this end, in this work the analysis of SSD paired with OFDMSPM is presented, thus presenting candidate schemes to meet these requirements. Additionally, the effect of receiver diversity schemes such as maximal ratio combining (MRC) on OFDM-SPM is also analyzed 1

The rest of the paper is organized as follows. In Section 2, we present the system model of OFDM-SPM-SSD and OFDM-SPM-MRC. The methodology and performance analysis is discussed in Section 3. Section 4 present the results and discussion, and Section 5 presents the conclusion of the work.

\section{SYSTEM MODEL}

In this section, a detailed description of the proposed model, its transmitter and receiver structures, and the considered multi-path channel model is provided.

\section{A. TRANSMITTER DESIGN}

The implementation of OFDM-SPM-SSD and the transmitter design of the proposed scheme are shown in Fig 1. In the transmitter diagram, the incoming bits are generated randomly and divided into two equal streams. For transmitting the first half of the total bits, traditional symbol modulation schemes are used, and the second half of bits is modulated using SPM [16]. The constellation is then rotated by applying some rotation angle, say $\Theta$. In general, if $S_{p}$ denotes the signal constellation for M-ary PSK modulation, then

$$
S_{p}=\left(S_{m}=e^{j 2 \pi \frac{m}{p}}: m=0,1,2, . . p-1\right) .
$$

When an anti-clockwise rotation is applied by an angle $\Theta$, then the rotated signal constellation can be represented by

$$
S_{p}^{\Theta}=\left(S_{m}=e^{j 2 \pi \frac{m}{p}+\Theta}: m=0,1,2, . . p-1\right) .
$$

\footnotetext{
${ }^{1}$ It should be noted that the security of the proposed scheme in this study can be met by integrating it with some advanced security approaches such as the ones proposed in [19], and [20]. In [19], an advanced novel small scale non-orthogonal communication technique utilizing physical layer security (PLS) for enhanced security and reliability for two users is proposed, where the proposed model uses the wireless channel characteristics to eliminates user interference as well as completely degrade the received signal at the eavesdropper's terminal. In [20], effective algorithms for enhancing physical layer security of OFDM systems are proposed.
} 
Since SSD combines rotation and interleaving system and this rotation constitutes one part of SSD implementation, null sub-carriers are added to the symbols for synchronization purposes and channel estimation [22]. Also, in null subcarriers, no data is transmitted, hence, they do not have any effect on transmitted power. Next, the coordinate interleaver is applied to the rotated symbols.

Coordinate interleaving is employed to ensure that the different constellation points experience an independent fading. Let $a_{i}$ and $b_{i}$ represent the I- and Q-components of the rotated constellation $S p^{\Theta}$, then $a_{i}=\left(a_{0}, a_{1}, \ldots \ldots a_{N-1}\right)$ and $b_{i}=$ $\left(b_{0}, b_{1}, \ldots \ldots b_{N-1}\right)$ represent the real and imaginary parts of $S_{p}^{\Theta}$, where $N$ represents the total number of symbols to be transmitted. We implement the coordinate interleaving just before performing the IFFT operation of traditional OFDM.

Hence, we give symbol sequence $x_{i}=a_{i}+j b_{i}$ at the input of the coordinate interleaver. The output of the coordinate interleaver can be represented as

$$
\hat{x}=a_{k}+j b_{m},
$$

such that $k, m \in(0,1,2 \ldots \ldots \ldots . N-1)$ with $k \neq m$. therefore, we can also express coordinate interleaver as $\hat{x}_{k}=\eta(x)$, where $\eta$ represents the coordinate interleaving function. Applying the IFFT operation, we get

$$
\hat{X}_{k}=\frac{1}{N} \sum_{n=0}^{N-1} \hat{x}_{k}(K) e^{j \frac{2 \pi}{N} n k} .
$$

Finally, a cyclic prefix (CP) is added to the symbol after IFFT because it mitigates the effect of inter-symbolinterference (ISI). In adding CP, the last $25 \%$ part of the OFDM symbol is added to the front of the symbol because ISI only affects the initial part of the OFDM symbol [20].

\section{B. CHANNEL MODEL}

The multi-path Rayleigh channel model with T-taps is considered. Let's represent our multi-path Rayleigh channel as $h$ such that

$$
h=\left[h_{0}, h_{1}, h_{2}, \ldots \ldots \ldots \ldots h_{t-1}\right] .
$$

Hence, the frequency domain response of the multi-path channel is given by

$$
h_{F}=F F T(h) .
$$

The signal received at the receiver is given as

$$
y=h \Theta \hat{X}_{k 1}+\eta_{0},
$$

where $\hat{X}_{k 1}$ represents the transmitted signal after adding $\mathrm{CP}$ in Eq.(4), $\Theta$ represents the convolution operation and $\eta_{0}$ is the AWGN noise with its corresponding signal to noise ratio (SNR) varying from $0 \mathrm{~dB}$ to $25 \mathrm{~dB}$.

\section{RECEIVER DESIGN}

The receiver design is implemented so that two different cases are investigated and studied. In the first case, OFDMSPM-SSD is implemented and for the second case, OFDMSPM-MRC is implemented.

\section{1) Receiver Design of OFDM-SPM-SSD}

For the first case, the receiver design is implemented as shown in Fig 2. The receiver implements the traditional stages of conventional OFDM such as CP removal and FFT followed by Zero Forcing equalization (ZFE). After FFT implementation, the signal at the receiver is given as

$$
\hat{Y}(n)=\sum_{n=0}^{N-1} y_{1}(k) e^{-j \frac{2 \pi}{N} n k} .
$$

Here, $y_{1}(k)$ is the signal obtained after the CP is removed from $y$ in Eq. (7). Then, coordinate de-interleaving is performed. The coordinate interleaving and de-interleaving processes are discussed in detail in section III. After the deinterleaving process, the demodulation process for M-PSK and SPM bits is done. The BER and throughput are simulated, computed and compared with the conventional OFDM.

\section{2) Receiver Design for OFDM-SPM-MRC}

As shown in Fig 3, OFDM-SPM-MRC diversity is implemented. Since MRC is a receiver diversity technique that employs two antennas at the receiver. Due to the use of two receiver antennas, two different channels are used to receive the OFDM-SPM signal. The channels in the case of MRC are defined as

$$
h_{1, r}=\left[h_{1,0}, h_{1,1}, h_{1,2}, \ldots \ldots \ldots \ldots h_{1, t-1}\right]
$$

and

$$
h_{2, r}=\left[h_{2,0}, h_{2,1}, h_{2,2}, \ldots \ldots \ldots \ldots . . . h_{2, t-1}\right]
$$

The corresponding frequency domain response of the channel is given as follows.

$$
\begin{aligned}
& h_{F, 1 r}=F F T(h 1, r) \\
& h_{F, 2 r}=F F T(h 2, r)
\end{aligned}
$$

The combiner for MRC combines the independent channels and the signals at the output of the MRC combiners is given by

$$
\hat{Y}(n)=a_{1}^{*} y_{1}(n)+a_{2}^{*} y_{2}(n)+\eta_{11},
$$

where $y_{1}(n)$ and $y_{2}(n)$ represent the signal received from the independent diversity branches, and $a_{1}^{*}$ and $a_{2}^{*}$ are the optimal weights of the MRC combiner [19]. The optimal weights of the MRC combiner can be found as:

$$
a_{1}^{*}=\frac{H_{F, 1 r}^{*}}{\left\|H_{F, 1 r}^{*}\right\|}
$$




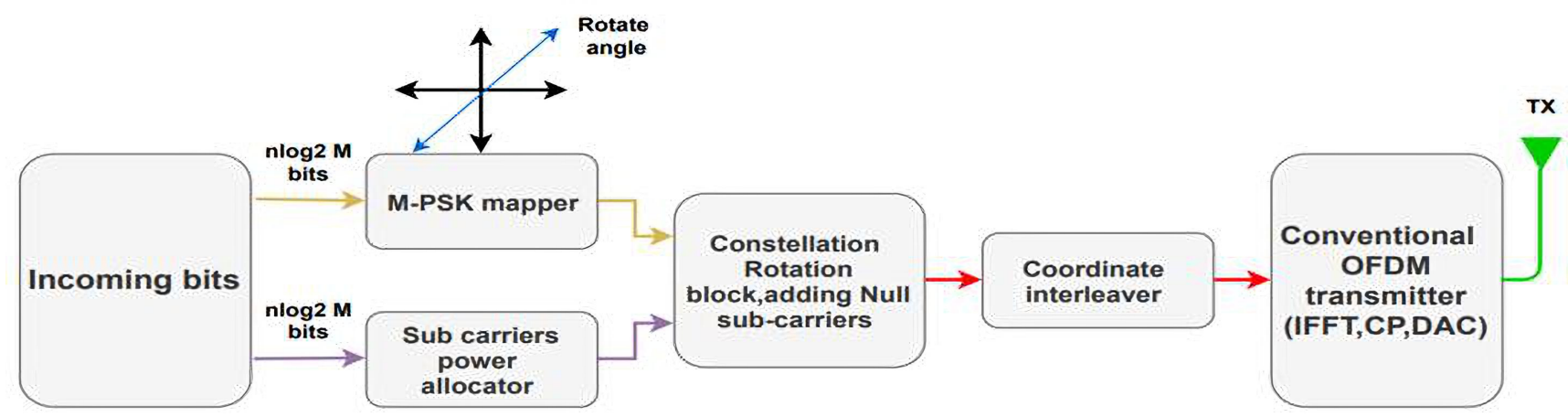

FIGURE 1: Transmitter design for OFDM-SPM-SSD.

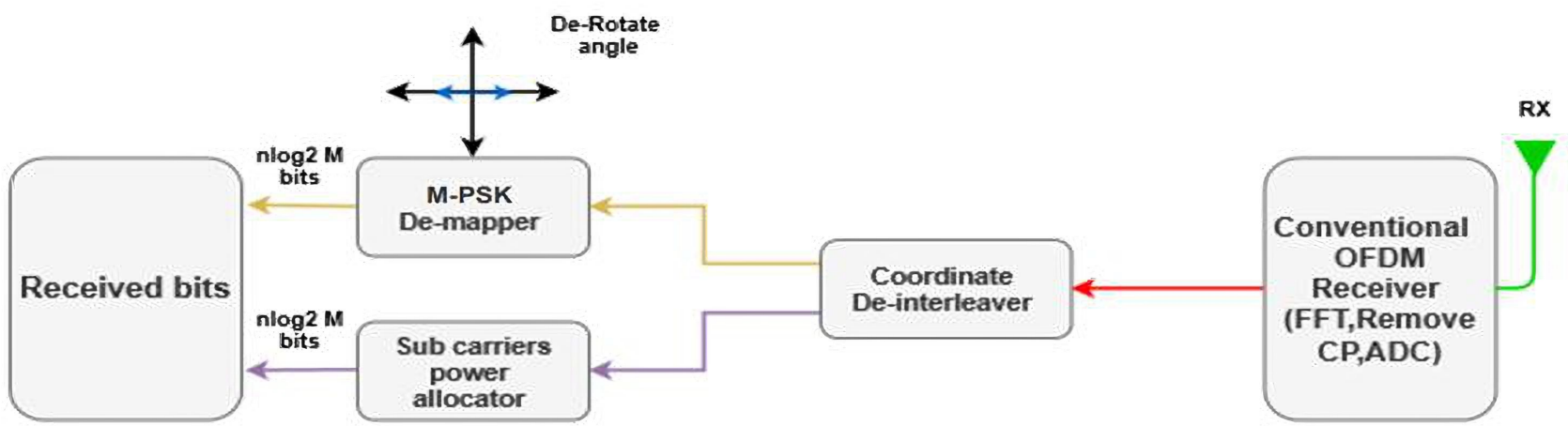

FIGURE 2: Receiver design of OFDM-SPM with SSD.

and

$$
a_{2}^{*}=\frac{H_{F, 2 r}^{*}}{\left\|H_{F, 2 r}^{*}\right\|}
$$

The output of the combiner is given to the demodulator for the detection of the transmitted bits of OFDM and the power bits transmitted through SPM separately.

\section{COORDINATE INTERLEAVING FOR SSD}

Coordinate interleaving is an important process for SSD and is defined by Eq. (3). The values of $k$ and $m$ are to be selected carefully as the performance of SSD highly depends on the characteristics of the interleaving method to be used and the delay profile of the channel. The coordinate interleaving is implemented as follows [15].

$$
\hat{x}=a_{k}+j b_{m}
$$

where

$$
\begin{aligned}
& m=K+M-L \quad \text { for } \quad 0<K \leq L \\
& m=K-L \quad \text { for } \quad L+1 \leq K<N
\end{aligned}
$$

The value of $L$ is selected in such a way that the correlation coefficient between the sub-carriers placed L-apart becomes zero. The correlation between the sub-carriers is then given as [25].

$$
R_{\hat{X}_{k}(L)}=E[\hat{X}(K) \hat{X}(K-L)]
$$

For a multipath fading channel having T-taps, we can represent the correlation coefficient of Eq. (16) as follows [15].

$$
P_{X}=\frac{1}{T}\left|\frac{\operatorname{Sin}\left(\frac{T}{N} L \pi\right)}{\operatorname{Sin}\left(\frac{1}{N} L \pi\right)}\right|
$$

Hence $P_{X}$ becomes zero when $\frac{T}{N} L$ is an integer and $\frac{L}{N}$ is not an integer. This is because when $\frac{L}{N}$ becomes an integer, the denominator term becomes zero. The values of $L$ should be between 1 to $N-1$. Fig. 4 shows how the correlation coefficient $P_{X}$ varies with sub-carrier distance and the values of $L$ are selected to satisfy Eq. (15) and (17). The rotation angle in the original constellation is also important. However, coordinate interleaving is not possible in BPSK but it is 


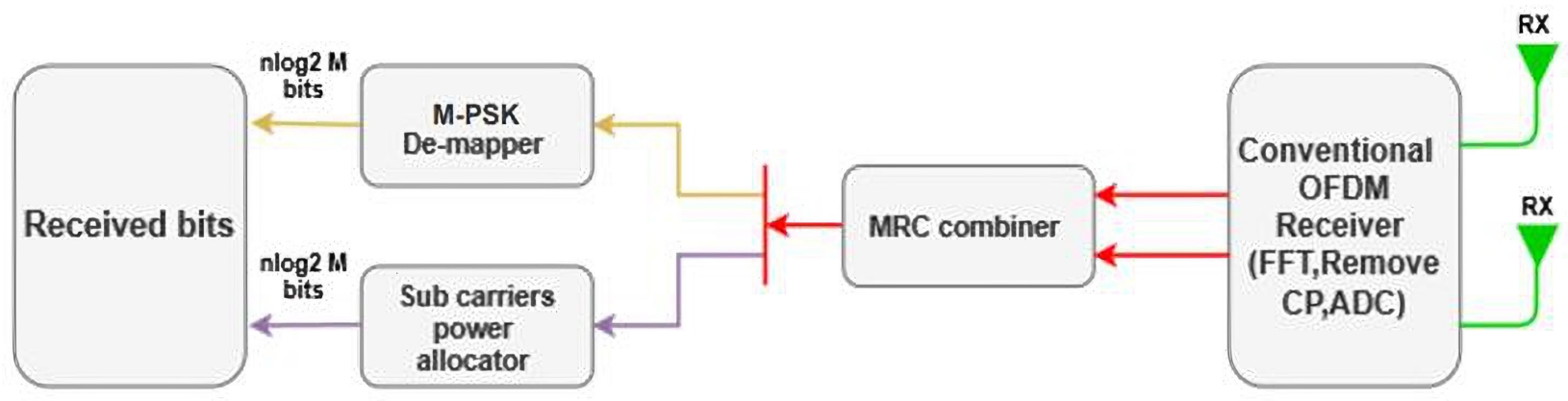

FIGURE 3: Receiver Design for OFDM-SPM with MRC diversity.

possible in rotated BPSK as the constellation becomes twodimensional after rotation, and coordinate interleaving can provide a good amount of diversity gain.

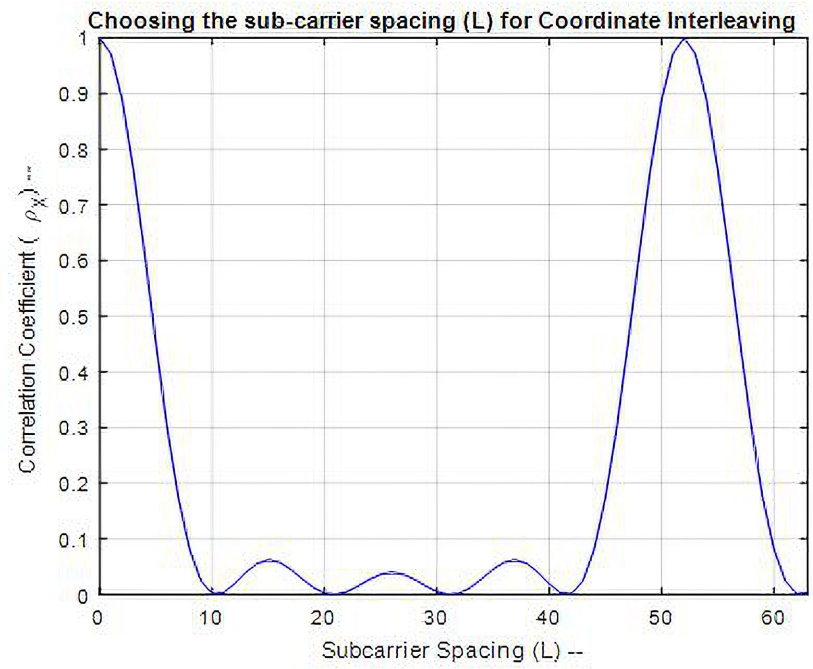

FIGURE 4: Subcarrier distance with correlation coefficient for coordinate interleaving in OFDM-SPM-SSD.

\section{RESULTS AND DISCUSSIONS}

In this section, simulation results of the OFDM-SPM-SSD and OFDM-SPM-MRC are presented and discussed. Two metrics, namely, the BER and throughput are analyzed and investigated over multi-path Rayleigh fading channels. Results for using different power policies such as power saving and power reassignment policies are also provided. Two cases are considered namely, the case with optimized power allocation and the other with non-optimized power allocation [21].

Each of these cases will be discussed separately. In the first sub-section, the results of OFDM-SPM-SSD-QPSK are shown taking into account all the cases of the power policies.
TABLE 1: Simulation settings for OFDM-SPM-QPSK-SSD.

\begin{tabular}{c|c}
\hline Modulation - Type & $S P M-S S D$ \\
\hline IFFT/FFT size & 64 \\
Number of Active Subcarriers & 52 \\
Cyclic prefix & 16 \\
Total number of sub-carriers & 64 \\
Rotation angle & $10^{0}$ \\
Number of inactive sub-carriers & 12 \\
Sub-carrier distance for Coordinate Interleaving (L) & 21 \\
For Power saving (SPM) [16] H & 1.35 \\
For Power saving (SPM) [16] L & 0.42 \\
Number of frames & $3 x 10^{4}$ \\
No of taps in multi-path channel & 5 \\
Multi path channel delay samples location & {$[0358]$} \\
Multi path channel tap power profile (dBm) & {$[0-8-17-21-25]$} \\
OFDM Bandwidth & $20 \mathrm{MHz}$ \\
OFDM symbol duration & $4 \mu \mathrm{sec}$ \\
Guard Interval & $0.8 \mu \mathrm{sec}$ \\
\hline
\end{tabular}

In the second sub-section, the results of OFDM-SPM-MRC are exhibited and discussed. Then a comparison between SSD and MRC for OFDM-SPM considering different power policies is given. Table 1 shows the simulation settings for BER and throughput performance calculation of ODFMSPM-SSD-QPSK.

\section{A. OFDM-SPM-QPSK-SSD}

The constellation points of OFDM-SPM-QPSK are shown in Fig 5. When SPM is applied to QPSK modulation in OFDM, the constellation of QPSK-SPM looks similar to 16-QAM. However, it has been shown and reported in the literature that QPSK-SPM significantly outperforms the 16QAM modulation [21]. The bits can be in error if either the bits modulated by conventional modulation QPSK or the bits modulated by the power modulation through sub-carriers are not detected correctly. The BER for both the QPSK and power bits is analyzed, and the average BER is computed.

\section{1) Power Saving Policy for OFDM-SPM-QPSK-SSD}

In the power saving policy, the power of the sub-carriers is saved to match the requirements of low power applications (e.g., IoT). This inherently results in a better power efficiency when compared to conventional OFDM. The $H$ and $L$ values 
for the power saving policy are found as $H=1.35$ and $L=0.4213$ [21], where $L$ and $H$ denote the low and high power levels of the sub-carriers, respectively. Fig 6 plots the BER of OFDM-SPM-QPSK-SSD in the case of using power saving policy, where half of the transmit power is saved in this case compared to conventional OFDM. It is observed that at a BER of $10^{-3}$, OFDM-SPM-QPSK-SSD has almost an $8 \mathrm{~dB}$ gain in SNR for achieving similar BER to that of OFDM-SPM-QPSK. Additionally, the OFDM-SPM-QPSKSSD scheme provides an improvement in the throughput at low SNR values compared to OFDM-SPM-QPSK, which is especially important for low power IoT applications. This is clearly demonstrated in Fig 7. It can also be understood that the effect of rotation on OFDM-SPM-QPSK-SSD is only marginal, whereas the core improvement in performance can be attributed to interleaving. This is because the constellation of OFDM-SPM-QPSK leaves very little room for angle rotation such that the points are still at a distinguishable distance.

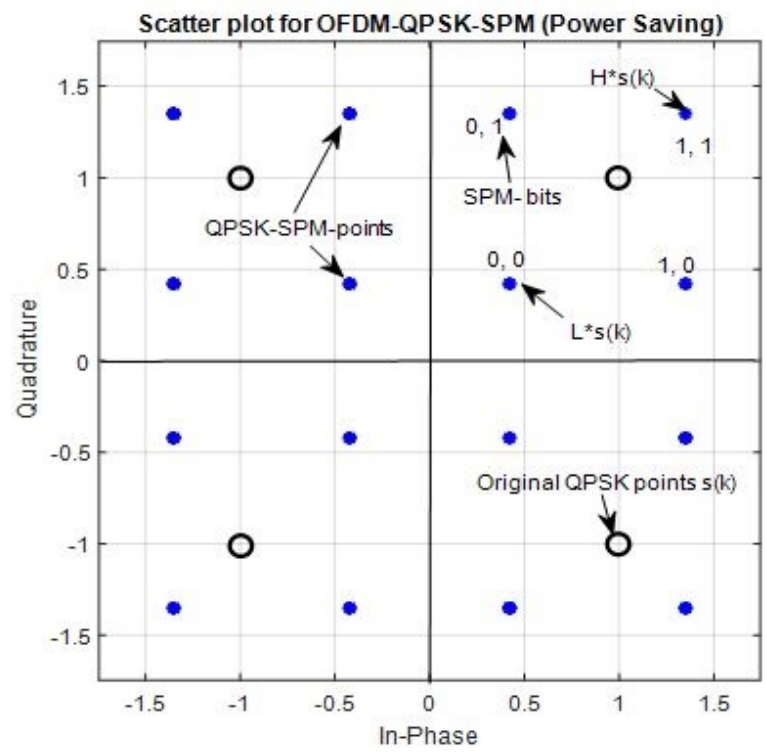

FIGURE 5: OFDM-QPSK-SPM constellation points without SSD.

\section{2) Power Reassignment for OFDM-SPM-QPSK-SSD}

In the reassignment power policy, we reassign the saved power as exhibited in the case of the power-saving policy to the sub-carriers.

- Power Reallocation - Non-Optimized Case:

In the case of non-optimized power reallocation, the saved power, which results from using the power difference in the subcarriers to convey additional information, is reassigned to the high-power sub-carrier while the lower power subcarriers are set to unity. The corresponding power levels are given as $H=1.732, L=1[21]$. The BER and throughput plots are exhibited in Fig 8 and Fig 9, respectively. It is observed that the power-reassignment policy provides a better SNR gain than the power-saving policy, thus better reliability performance as demonstrated from the BER curves in Fig 8 . But the SNR gain is almost constant in the throughput SNR range.

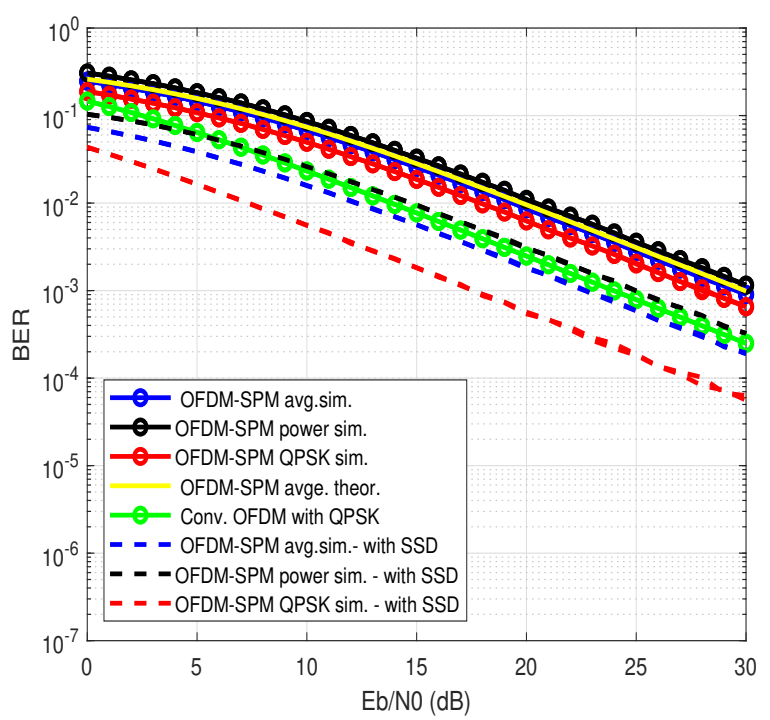

FIGURE 6: Comparing BER for OFDM-SPM-QPSK with and without SSD (power saving policy)

- Power Reallocation - Optimized Case: In this case, the saved power is reassigned to the sub-carriers to optimize the average BER and make it as low as possible. The optimal high and low power levels that can achieve the best average BER performance are obtained as $H=1.91$, and $L=0.56$ [21].

This optimization is done under a constraint that ensures that the average energy of an OFDM sub-carrier in OFDMSPM cannot exceed that of a sub-carrier in conventional OFDM. Fig. 10 and 11 show the BER results in the case of an optimized power reallocation policy. We observe that although the results of BER are better in the case of power reallocation policy than in power saving policy, but as can be seen from Fig. 10 and Fig 11, not much variation is found in the BER in the cases of optimized and non-optimized power reallocation.

The similar inference is observed in the case of throughput. When comparing the OFDM-SPM-QPSK-SSD with normal OFDM-SPM-QPSK, it's always observed that OFDM-SPMQPSK-SSD results in better performance in terms of both BER and throughput in all the cases.

\section{B. OFDM-SPM-QPSK-MRC}

In this section, the simulation results for OFDM-SPMQPSK-MRC are provided and compared with OFDM-SPM- 


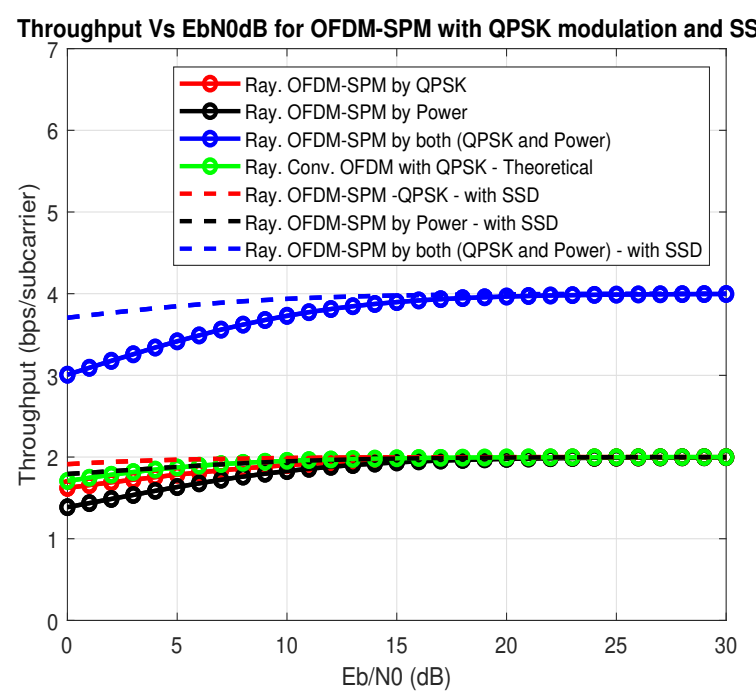

FIGURE 7: Comparison of throughput of OFDM-SPMQPSK with and without SSD with power-saving SPM

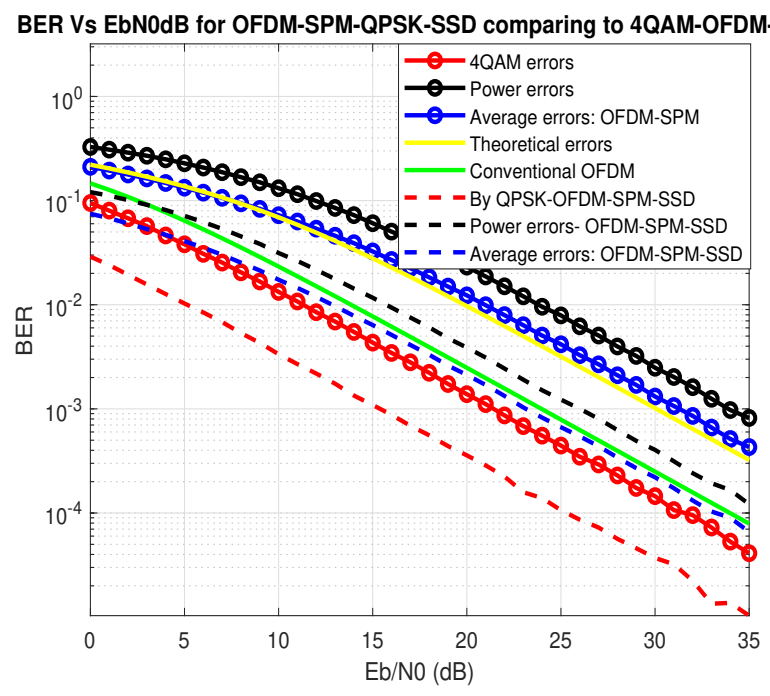

FIGURE 8: Comparing BER for OFDM-SPM-QPSK with and without SSD (power reallocation - non-optimized)

QPSK-SSD. MRC involves the combination of all receive antennas as such the performance is improved at the cost of increased detection complexity due to combining signals from multiple antennas. Results taking different power policies are provided as considered in the previous sub-section.

\section{1) Power Saving Policy for OFDM-SPM-QPSK-MRC}

Fig. 12 shows the BER comparison between OFDM-SPMQPSK without SSD, with SSD, and with MRC for power saving SPM policy [21]. It's observed that MRC provides an improvement over conventional the OFDM-SPM- QPSK due to utilizing receive diversity. MRC achieves 2-3 dB gain

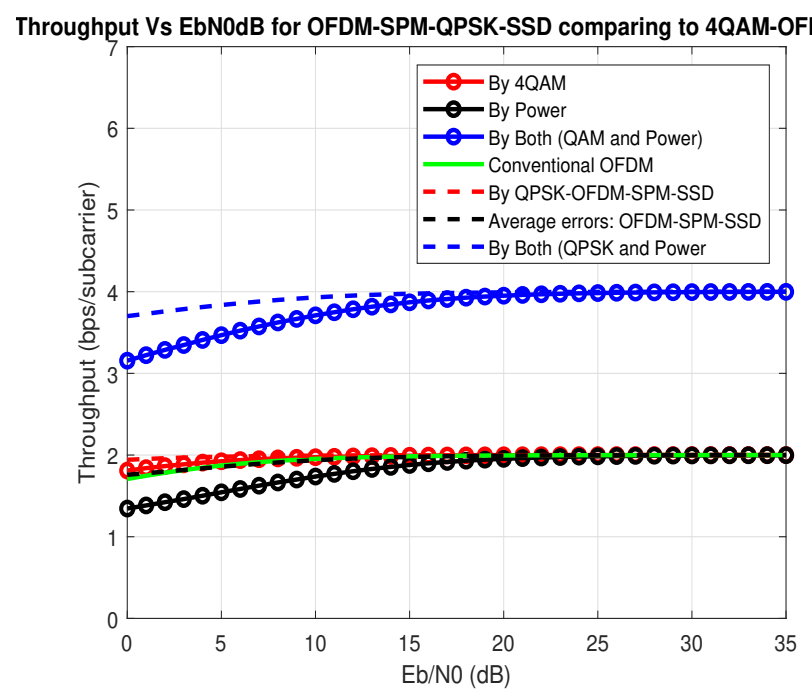

FIGURE 9: Comparison of throughput for OFDM-SPMQPSK with and without SSD with power-reallocation (nonoptimized)

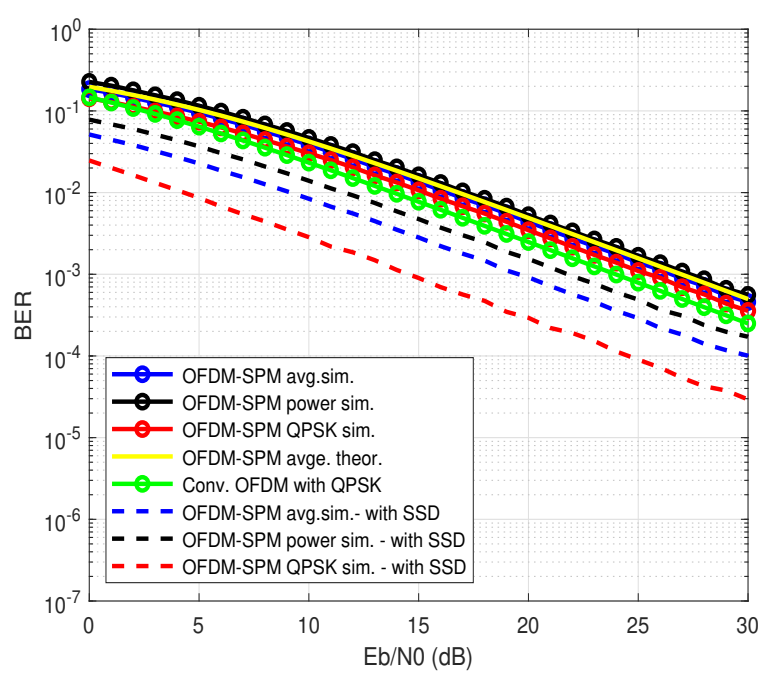

FIGURE 10: Comparing BER of OFDM-SPM-QPSK with and without SSD (power reallocation -optimized case)

in SNR at BER of $10^{-2}$ over conventional OFDM-SPMQPSK. The gain is expected due to the combination of two independent channels but at the cost of additional hardware by using two antennas and an additional combiner. However, SSD provides performance improvement without using extra power or bandwidth. While comparing the BER of SSD and MRC in Fig. 12, it is found out that SSD archives a gain of 4-5 dB SNR.

Furthermore, it can be stated that although MRC and SSD exploit diversity to enhance the BER, SSD shows better performance results. This is because SSD exploits the inherent 


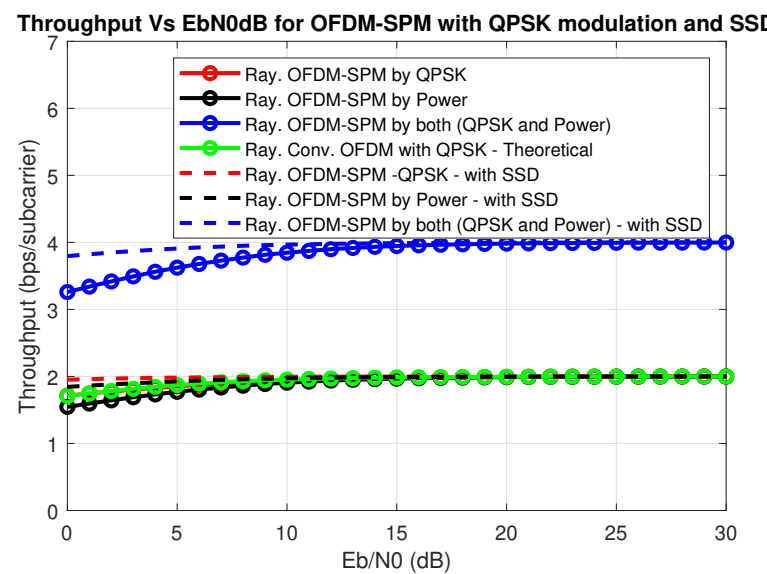

FIGURE 11: Comparison of Throughput for OFDM-SPMQPSK with and without SSD with power-reallocation (optimized)

diversity existing in the orthogonality of signal space [14]. Fig.13 plots the throughput comparison of MRC and SSD. It is observed that SSD also provides an improvement in throughput over MRC.

The throughput improvement is observed at low SNR, which is particularly important because, at lower SNR levels, the transmitted data is more prone to being received in error as appose to where the signal power increase provides an obvious strength in the overall robustness of the transmitted data to noise and other factors. To better analyze the effect of the rotation on the scheme, we move to the lower constellation points, which provide a sufficient number of distinct components of the signal space in the constellation diagram of OFDM-SPM,for example at SNR $5 \mathrm{~dB}$ the throughput is equal to 1.6 for convenience OFDM-SPM , as when OFDMSPM -SSD was applied the throughput has been increased.

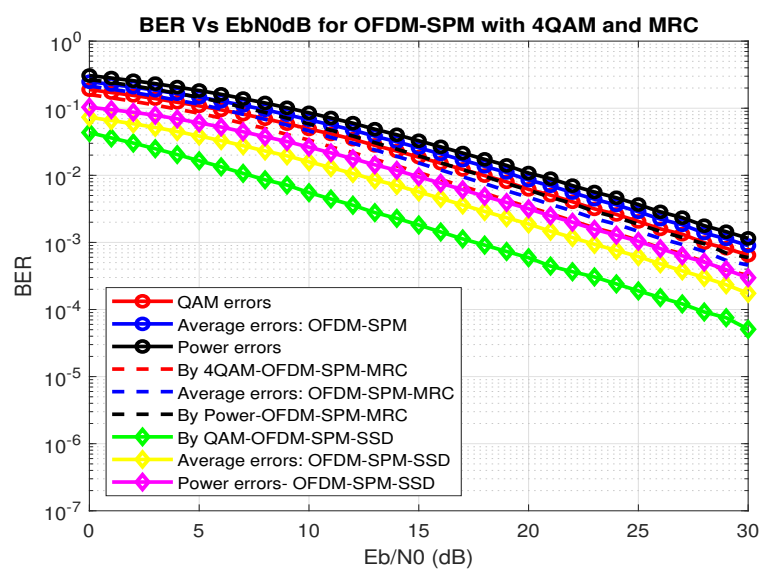

FIGURE 12: Comparison of OFDM-SPM-QPSK-MRC and OFDM-SPM-QPSK-SSD (power-saving)

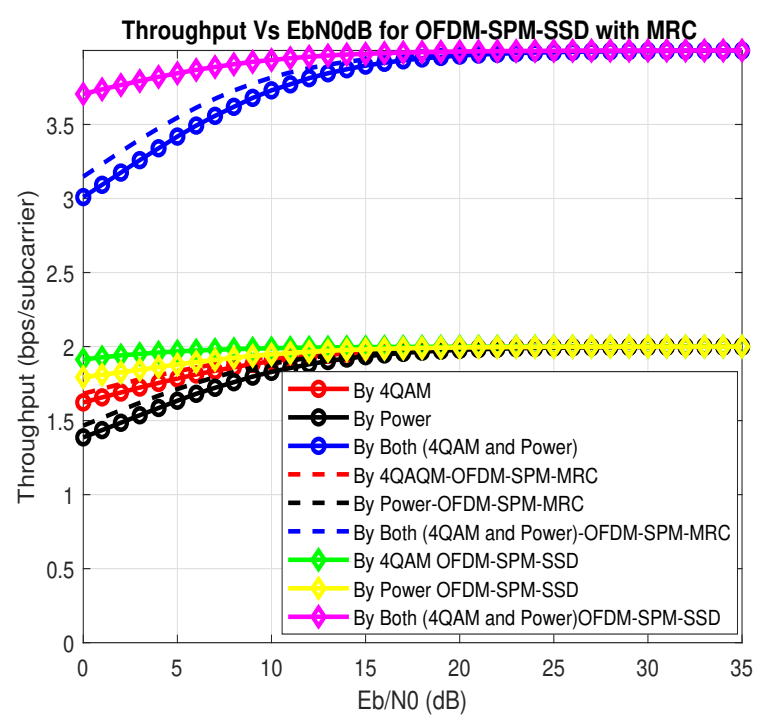

FIGURE 13: Throughput Improvement in OFDM-SPMQPSK-MRC and SSD (power-saving)

\section{2) Power Reassignment for OFDM-SPM-QPSK-MRC}

The power reassignment policy is considered using regards to different cases.

- Power Reassignment - Non-Optimized Case:

In this case, the power levels of the sub-carriers of SPM are taken as $H=1.732$ and $L=1$. Fig. 14 and Fig.15 show the BER and throughput for MRC and SSD, in the case of non-optimized power levels for SPM.

A significant performance enhancement is observed for OFDM-SPM-QPSK-MRC as can be shown in Fig. 14 and Fig. 15; however, it is still evident that OFDM-SPM-QPSKSSD outperforms OFDM-SPM-QPSK-MRC. Similarly, although an improvement in the throughput is observed in the case of using MRC, SSD shows to provide better diversity which is evident from the throughput results.

- Power -Reassignment - Optimized Case:

The results of power-optimized OFDM-SPM are given in Fig. 16 and Fig. 17.

It is observed that OFDM-SPM-QPSK-MRC shows a marginal degradation in the BER whereas OFDM-SPMQPSK-SSD shows more improvement in the BER. 


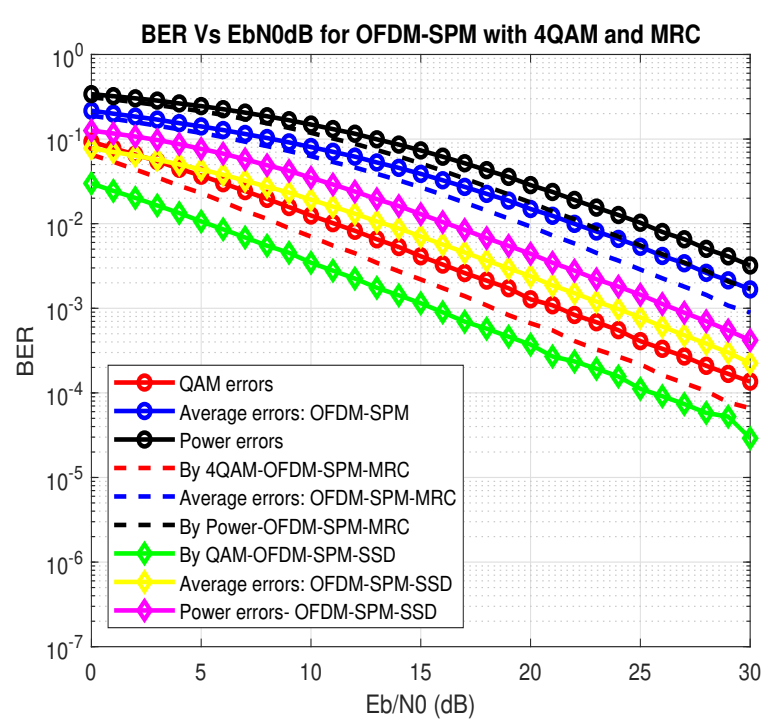

FIGURE 14: Comparison of OFDM-SPM-QPSK-MRC and OFDM-SPM-QPSK-SSD (power-reassignment-non- optimized)

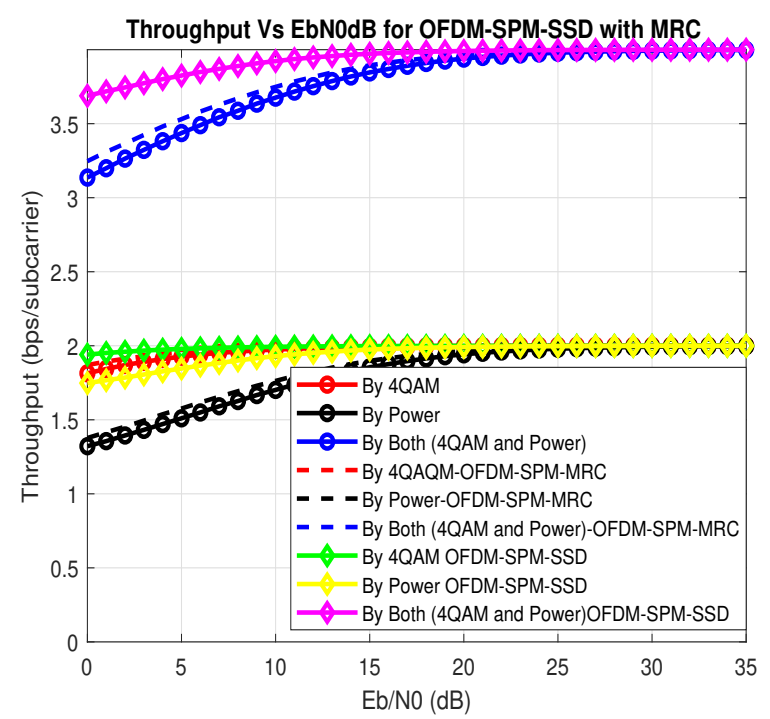

FIGURE 15: Throughput Improvement in OFDM-SPMQPSK-MRC and SSD (power-reassignment-non- optimized)

\section{EFFECT OF ROTATION OF CONSTELLATION IN OFDM-SPM-BPSK-SSD}

To study the effect of constellation rotation, it was implemented to a lower modulation scheme in SPM. Coordinate interleaving along with the rotation of constellation was implemented for OFDM-SPM-BPSK-SSD as given in [10].

It is observed that rotation of constellation results in an improvement in BER in the case of BPSK. Another point here worth noting is that coordinate interleaving cannot be implemented to conventional BPSK symbol modulation; this is because it inherently has only real constellation points. The coordinate interleaving can only be implemented in rotated BPSK constellation.

Fig. 18 shows the effect of angle rotation on BER for OFDM-SPM-BPSK-SSD. We observe that the small rotation angle in the constellation of QPSK improves the BER.

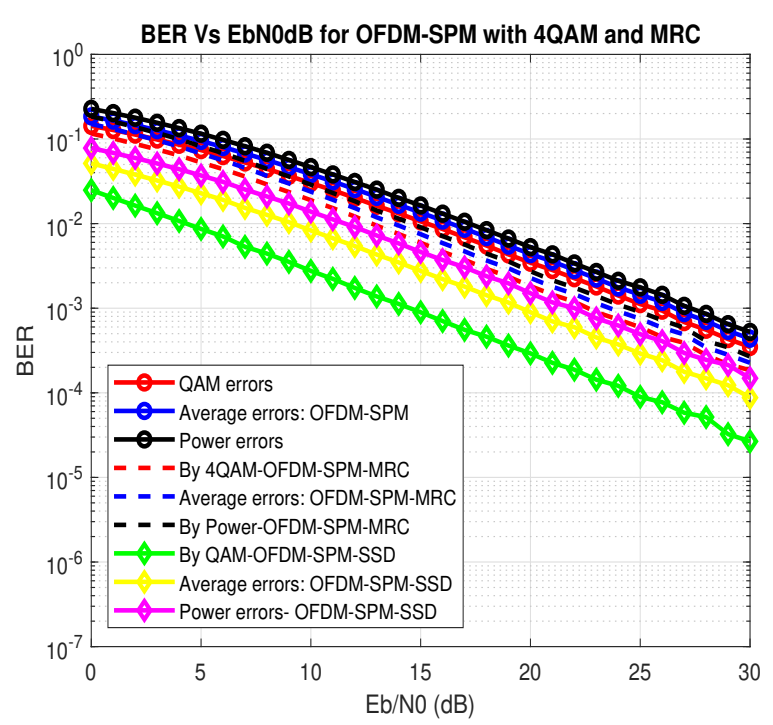

FIGURE 16: Comparison of OFDM-SPM-QPSK-MRC and OFDM-SPM-QPSK-SSD (power-reassignment - optimized.)

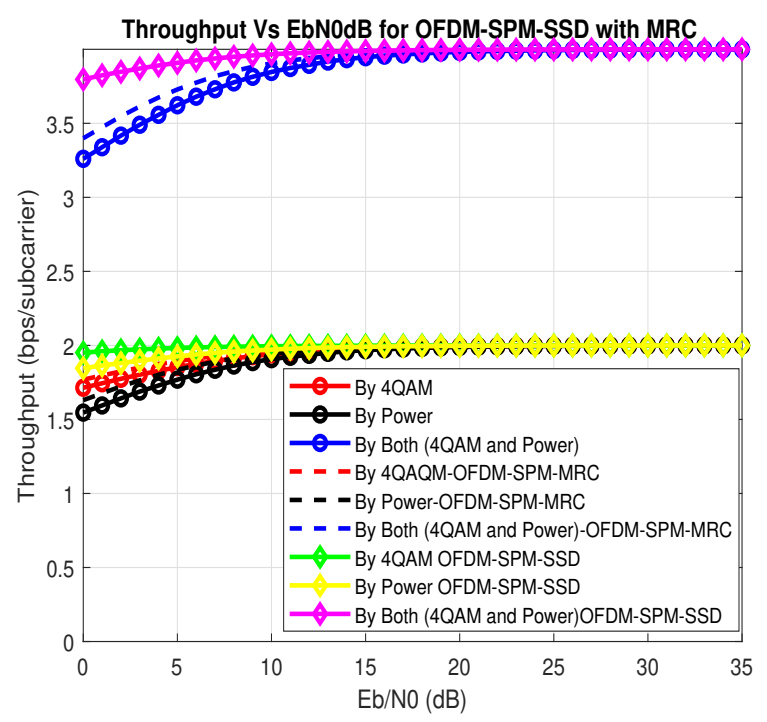

FIGURE 17: Throughput Improvement in OFDM-SPMQPSK-MRC and SSD (power-reassignment - optimized) 


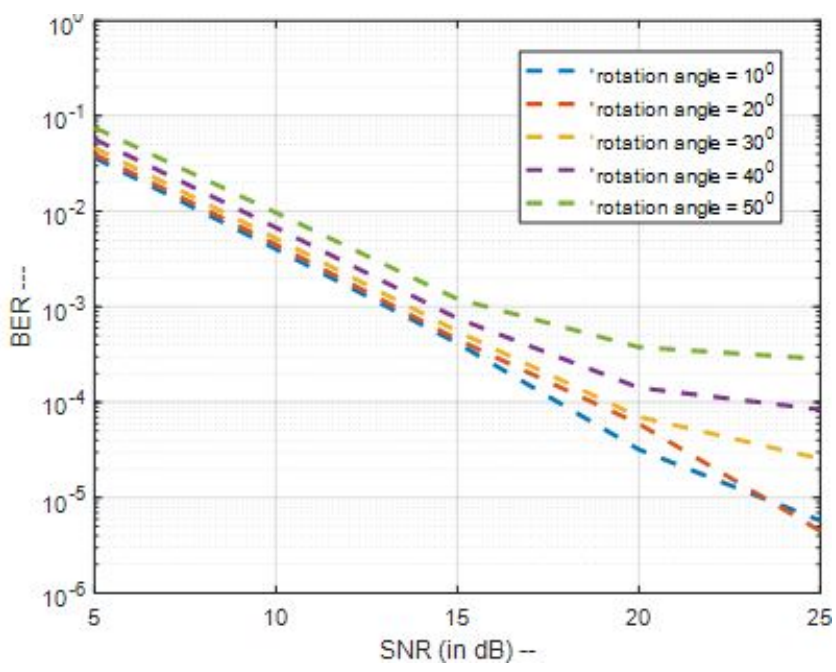

FIGURE 18: Effect of rotation on BER of OFDM-SPMBPSK-SSD.

\section{CONCLUSION AND FUTURE SCOPE}

In this paper, the performance of OFDM-SPM is studied when paired with SSD. SSD provides diversity in signal space by exploiting the orthogonality of the signal space and hence it is different from receiver diversity such as MRC. OFDM-SPM-QPSK with SSD were implemented and the results of BER and throughput were compared with the OFDM-SPM-QPSK-MRC technique. Different power policies for OFDM-SPM were considered such as power-saving and power-reassignment. Also, the effect of constellation rotation on OFDM-SPM-BPSK-SSD was studied.

It was concluded that when SSD is applied to OFDMSPM-QPSK, the effect of constellation rotation is only marginal and the improvement in BER is mainly due to interleaving. Moreover, OFDM-SPM-QPSK-SSD is shown to provide a considerable improvement in the BER and throughput over the traditional OFDM-SPM-QPSK and OFDM-SPM-QPSK-MRC. When the results were compared for different power policies, it was found that the BER of OFDM-SPM-QPSK-SSD is improved more in the case of using power-reassignment policy compared to the case of using power saving policy. Also, it was observed that the difference in BER is only marginal when we apply the optimized power reassignment and non-optimized power reassignment for OFDM-SPM-QPSK-SSD.

\section{REFERENCES}

[1] A. Jaradat, J. M. Hamamreh, H. Arslan, "Modulation Options for OFDMBased Waveforms: Classification, Comparison, and Future Directions," IEEE Access, 7(1), 17263-17278, Feb. 2019.

[2] A. M. Jaradat, J. M. Hamamreh and H. Arslan, "OFDM With Hybrid Number and Index Modulation," in IEEE Access, vol. 8, pp. 55042-55053, 2020.

[3] J. M. Hamamreh, Z. E. Ankarali, and H. Arslan, "CP-Less OFDM with Alignment Signals for Enhancing Spectral Efficiency, Reducing Latency, and Improving PHY Security of 5G and Beyond Services," in IEEE Access, vol. 6, pp. 63649-63663, 2018.

[4] J. M. Hamamreh, E. Basar, and H. Arslan, "OFDM-subcarrier index selection for enhancing security and reliability of 5G URLLC services," IEEE Access, vol. 5, pp. 25 863-25 875, 2017.

[5] E. Başar, "OFDM With Index Modulation Using Coordinate Interleaving,' in IEEE Wireless Communications Letters, vol. 4, no. 4, pp. 381-384, Aug. 2015, doi: 10.1109/LWC.2015.2423282.

[6] A. Hajar, J. M. Hamamreh, M. Abewa and Y. Belallou, "A Spectrally Efficient OFDM-Based Modulation Scheme for Future Wireless Systems," 2019 Scientific Meeting on Electrical-Electronics Biomedical Engineering and Computer Science (EBBT), Istanbul, Turkey, 2019, pp. 1-4, doi: 10.1109/EBBT.2019.8742049.

[7] Y. Belallou, J. M. Hamamreh and A. Hajar, "OFDM-Subcarrier Power Modulation with two-dimensional signal constellations," 2019 Innovations in Intelligent Systems and Applications Conference (ASYU), Izmir, Turkey, 2019, pp. 1-6, doi: 10.1109/ASYU48272.2019.8946346.

[8] Abewa, M., Hamamreh, J. M. (2020). Non-coherent OFDM-Subcarrier Power Modulation for Low Complexity and High Throughput IoT Applications. RS Open Journal on Innovative Communication Technologies.

[9] Hajar, A., Hamamreh, J. M. (2020). The Generalization of Orthogonal Frequency Division Multiplexing With Subcarrier Power Modulation to Quadrature Signal Constellations. RS Open Journal on Innovative Communication Technologies.

[10] J. M. Hamamreh, Abdulwahab Hajar, and Mohamedou Abewa, "Orthogonal Frequency Division Multiplexing With Subcarrier Power Modulation for Doubling the Spectral Efficiency of 6G and Beyond Networks." in Transactions on Emerging Telecommunications Technologies, 2020.

[11] H. C. Mora, N. Orozco Garzón, F. A. García and C. de Almeida, "On the Bit Error Rate of OFDMA Employing Short Cyclic Prefix and Maximal Ratio Combining," 2019 IEEE Colombian Conference on Communications and Computing (COLCOM), Barranquilla, Colombia, 2019, pp. 1-6, doi: 10.1109/ColComCon.2019.8809120.

[12] Bo-Seok Seo and Seong-Gon Choi, "Maximum ratio combining for OFDM systems with cochannel interference," 2006 Digest of Technical Papers International Conference on Consumer Electronics, Las Vegas, NV, 2006, pp. 365-366, doi: 10.1109/ICCE.2006.1598462.

[13] S. Nandi, A. Nandi and N. N. Pathak, "Performance analysis of Alamout STBC MIMO OFDM for different transceiver system," 2017 Internationa Conference on Intelligent Sustainable Systems (ICISS), Palladam, 2017, pp. 883-887, doi: 10.1109/ISS1.2017.8389305.

[14] J. Boutros and E. Viterbo, "Signal space diversity: a power- and bandwidth-efficient diversity technique for the Rayleigh fading channel," in IEEE Transactions on Information Theory, vol. 44, no. 4, pp. 1453-1467, July 1998, doi: 10.1109/18.681321.

[15] S. Özyurt and O. Kucur, "Performance of OFDM With Signal Space Diversity Using Subcarrier Coordinate Interleaving," in IEEE Transactions on Vehicular Technology, vol. 67, no. 10, pp. 10134-10138, Oct. 2018, doi 10.1109/TVT.2018.2864645.

[16] J. C. Inácio, B. F. Uchôa-Filho and D. Le Ruyet, "Exploiting Signal Space Diversity in OFDM With Grouped Subcarriers: Going Beyond Subcarrier Index Modulation," in IEEE Wireless Communications Letters, vol. 7, no. 4, pp. 650-653, Aug. 2018, doi: 10.1109/LWC.2018.2807446.

[17] C. I. Frison, H. C. Mora, C. Almeida, "Optimum rotation parameters for single and multiuser signal space diversity employing spatial diversity," AEU - International Journal of Electronics and Communications, vol. 107, pp. 275-281, 2019, doi: 10.1016/j.aeue.2019.05.032.

[18] N. F. Kiyani, J. H. Weber, A. G. Zajic and G. L. Stuber, "Performance Analysis of a System using Coordinate Interleaving and Constellation Rotation in Rayleigh Fading Channels," 2008 IEEE 68th Vehicular Technology Conference, Calgary, BC, 2008, pp. 1-5, doi: 10.1109/VETECF.2008.219.

[19] Lemayian, J. P., Hamamreh, J. M. (2020). A Novel Small-Scale Nonorthogonal Communication Technique Using Auxiliary Signal Superposition with Enhanced Security for Future Wireless Networks. RS Open Journal on Innovative Communication Technologies.

[20] H. M. Furqan, J. M. Hamamreh, and H. Arslan, "Adaptive OFDM-IM for Enhancing Physical Layer Security and Spectral Efficiency of Future Wireless Networks," Wireless Communications and Mobile Computing by Hindawi and Wiley, vol. 2018, Article ID 3178303, 16 pages, 2018. doi: $10.1155 / 2018 / 3178303$

[21] J. M. Hamamreh, and A. Hajar, "The Generalization of Orthogonal Frequency Division Multiplexing with Subcarrier Power Modulation to 
Quadrature Signal Constellations", RS Open Journal on Innovative Communication Technologies, 1 (1), 2020, doi: 10.21428/03d8ffbd.4948e89e.

[22] P. Zetterberg, M. L. Nordenvaad and B. Nilsson, "Synchronization of OFDM with null subcarriers in channels with significant ICI," OCEANS 2017 - Aberdeen, Aberdeen, 2017, pp. 1-5, doi: 10.1109/OCEANSE.2017.8084727.

[23] J. P. Singh and S. Singh, "Implementation of OFDM and other multicarrier modulations on SDR," 2016 IEEE International Conference on Signal Processing, Communication, Power and Embedded System (SCOPES), Paralakhemundi, India, 2016, pp. 1937-1940.

[24] A. M. S. Abdelgader, L. Wu, "Design of a Multi-order Frequency Diversity Approach," Mathematical Problems in Engineering, Hindawi, Vol. 2015, doi: $10.1155 / 2015 / 291625$

[25] Xiaolong Zhu and Jinyin Xue, "On the Correlation of Subcarriers in Grouped Linear Constellation Precoding OFDM Systems Over Frequency Selective Fading," 2006 IEEE 63rd Vehicular Technology Conference, Melbourne, Vic., 2006, pp. 1431-1435, doi: 10.1109/VETECS.2006.1683071.

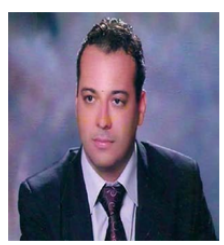

MOHAMMED HIJAZI received the B.Sc. degree in Electronics Engineering from Princess Sumaya University, Jordan, in 2005.

$\mathrm{He}$ is the co-author of Signal Space Diversityassisted OFDM-SPM, and has worked with Professor Jehad M. Hamamreh on the topic of OFDM-SPM-SSD. He is currently completing his master degree in the field of electrical and computer engineering at Antalya Bilim University in Antalya, Turkey.

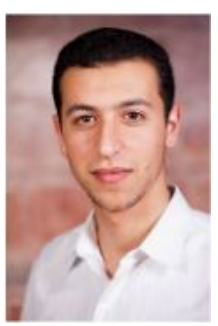

JEHAD M. HAMAMREH received the B.Sc. degree in electrical and telecommunication engineering from An-Najah University, Nablus, in 2013, and the Ph.D. degree in electrical-electronics engineering and cyber systems from Istanbul Medipol University, Turkey, in 2018. He was a Researcher with the Department of Electrical and Computer Engineering, Texas A and M University. He is currently an Assistant Professor with the Electrical and Electronics Engineering Department, Antalya International (Bilim) University, Turkey.

His current research interests include wireless physical and MAC layers security, orthogonal frequency-division multiplexing multiple-input multiple-output systems, advanced waveforms design, multi-dimensional modulation techniques, and orthogonal/non-orthogonal multiple access schemes for future wireless systems. He is a regular investigator and referee for various scientific journals as well as a TPC Member for several international conferences. He can be reached via e-mail: jehad.hamamreh@gmail.com // web: https://sites.google.com/view/wislab. 